\title{
Pulmonary Tumor Thrombotic Microangiopathy Caused by Urothelial Carcinoma Expressing Vascular Endothelial Growth Factor, Platelet-derived Growth Factor, and Osteopontin
}

\author{
Yasushi Wakabayashi ${ }^{1}$, Mai Iwaya ${ }^{2}$, Mayo Akita ${ }^{1}$, Wataru Takeuchi ${ }^{1}$, \\ Kyohei Yamazaki ${ }^{1}$ and Akihiro Iijima ${ }^{1}$
}

\begin{abstract}
Pulmonary tumor thrombotic microangiopathy (PTTM) is a fatal cancer-related pulmonary complication. It is generally caused by gastric adenocarcinoma, and several molecules produced by tumor cells are reported to play important roles in its pathogenesis. We herein report an autopsy case of PTTM caused by urothelial carcinoma. Vascular endothelial growth factor (VEGF), platelet-derived growth factor (PDGF), and osteopontin were found to be expressed in both the primary tumor cells and metastatic cells in the PTTM lesions. These findings implicate the possible involvement of VEGF, PDGF, and osteopontin in the pathogenesis of PTTM caused by urothelial carcinoma.
\end{abstract}

Key words: pulmonary tumor thrombotic microangiopathy, urothelial carcinoma, vascular endothelial growth factor, platelet-derived growth factor, osteopontin

(Intern Med 55: 651-656, 2016)

(DOI: 10.2169/internalmedicine.55.5758)

\section{Introduction}

Pulmonary tumor thrombotic microangiopathy (PTTM) is known to be a rare and fatal pulmonary complication characterized by progressive dyspnea and pulmonary hypertension in patients with metastatic carcinoma. According to the first report by von Harvey et al., PTTM was observed in $3.3 \%(21 / 630)$ of the autopsy cases with malignant tumors (1). With respect to the underlying malignancy, the most frequent organ and histological type are reported to be the stomach and adenocarcinoma, respectively $(1,2)$, and only 3 cases of PTTM associated with urothelial carcinoma have been reported in the English literature $(1,3,4)$. We herein present a case of PTTM associated with urothelial carcinoma, including an immunohistochemical analysis that revealed important clues for the pathogenesis of PTTM caused by urothelial carcinoma.

\section{Case Report}

A 77-year-old man was diagnosed with ureteral carcinoma of the left ureter and underwent total nephroureterectomy with partial cystectomy. The pathological findings of the surgical specimens indicated urothelial carcinoma with squamous differentiation (G3) that had invaded into the subserosa (pT3) with lymphatic involvement. Moreover, carcinoma in situ was detected on the surgical margin of the urinary bladder. Although additional therapy was recommended, the patient chose to forgo additional treatment. Five years after the surgery, he was admitted to our hospital due to dyspnea on exertion.

At this admission, the patient's blood pressure was 160/ $90 \mathrm{mmHg}$ and his heart rate was 82 beats per minute. His percutaneous oxygen saturation was $92 \%$ on room air. Laboratory tests revealed a decrease in the platelet count $(111,000 / \mu \mathrm{L})$ and an increase in lactate dehydrogenase $(522$

${ }^{1}$ Department of Internal Medicine, Nagano Prefectural Kiso Hospital, Japan and ${ }^{2}$ Department of Laboratory Medicine, Shinshu University Hospital, Japan

Received for publication May 12, 2015; Accepted for publication June 25, 2015

Correspondence to Dr. Yasushi Wakabayashi, 99098yw@jichi.ac.jp 
b

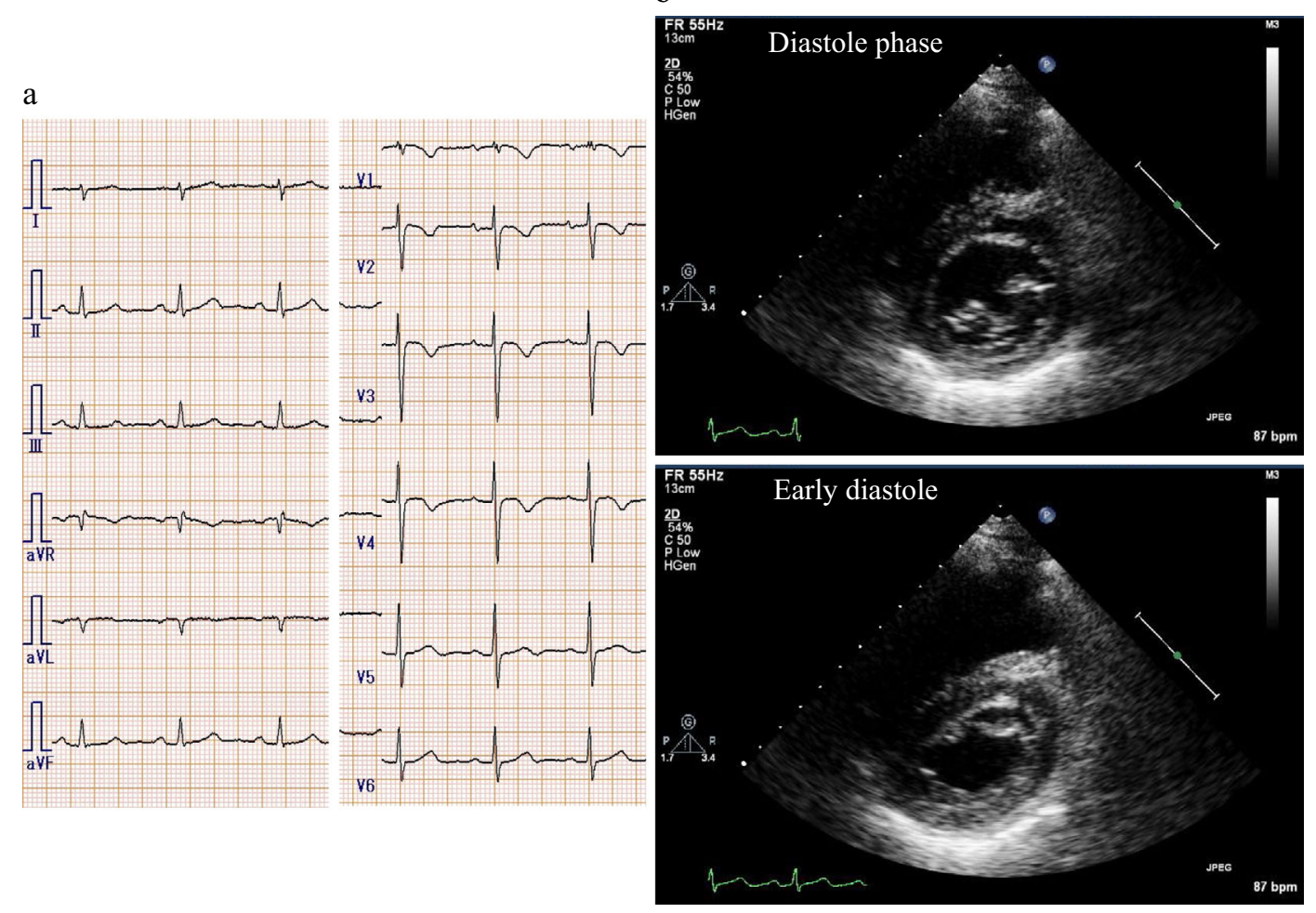

Figure 1. Electrocardiography and transthoracic echocardiography. a) Electrocardiography showed right axis deviation and negative $T$ waves in V1, V2, V3, and V4. b) Transthoracic echocardiography revealed a D-shape appearance of the left ventricular chamber in early diastole.

IU/L), fibrin degradation product $(18.2 \mu \mathrm{g} / \mathrm{mL})$, and $\mathrm{D}$ dimer $(6.5 \mu \mathrm{g} / \mathrm{mL})$. His brain natriuretic peptide level was $92.98 \mathrm{pg} / \mathrm{mL}$. The results did not suggest any collagen diseases that would lead to pulmonary hypertension. His arterial blood gas examination when breathing room air showed hypoxia with respiratory alkalosis $(\mathrm{pH}, 7.47$; arterial carbon dioxide partial pressure, $29.0 \mathrm{mmHg}$; arterial oxygen partial pressure, $56.0 \mathrm{mmHg}$; and bicarbonate, $20.8 \mathrm{mEq} / \mathrm{L}$ ). Chest radiography showed dilated pulmonary arteries without congestion. Electrocardiography showed right axis deviation and negative $\mathrm{T}$ wave in $\mathrm{V} 1, \mathrm{~V} 2, \mathrm{~V} 3$, and $\mathrm{V} 4$, suggesting right ventricular overload (Fig. 1a). Transthoracic echocardiography demonstrated a dilated right atrium and ventricle, and a D-shape of the left ventricular chamber in early diastole (Fig. 1b). The tricuspid regurgitation pressure gradient was $110 \mathrm{mmHg}$, suggesting pulmonary hypertension. Contrastenhanced computed tomography showed no thrombi or tumor emboli in the pulmonary arteries. However, lymphadenopathy of the left supraclavicular, para-aortic, and left pelvic lymph nodes was observed, suggesting multiple lymph node metastases (Fig. 2). A supraclavicular lymph node biopsy was performed and urothelial carcinoma with squamous differentiation was found. Thus, the patient was diagnosed with recurrent ureteral cancer with lymph node metastasis. Although we suspected PTTM, we could not make a definitive diagnosis because the patient refused an invasive lung biopsy procedure. He also refused intensive chemotherapy and was discharged after 28 days, using home oxygen therapy.

Twenty-nine days after he was discharged, he was readmitted due to general fatigue and progressive dyspnea, and multiple organ failure was confirmed. Respiratory failure progressed rapidly and he died several hours after admission.

With the approval of the patient's family, a postmortem autopsy was performed. No macroscopic thrombi or tumor emboli were detected in the pulmonary arteries or their main branches, although the right ventricle was dilated. Furthermore, we could not find tumor formation in the lung parenchyma. However, a microscopic examination of the lungs revealed multiple tumor emboli of the urothelial carcinoma with squamous differentiation in the small pulmonary arteries. Marked intimal fibrocellular proliferation and clot formation were also observed in the small pulmonary arteries, and these findings were consistent with the typical features of PTTM (Fig. 3). A number of metastases were detected in the left supraclavicular, para-aortic, and left pelvic lymph nodes. The final diagnosis after the autopsy was PTTM caused by urothelial carcinoma of recurrent ureteral cancer.

We performed immunohistochemical staining of the primary tumor cells resected 5 years previously and metastatic cells in the PTTM lesions using antibodies against vascular endothelial growth factor (VEGF) (JH121; dilution 1:500, EMD Millipore, Billerica, USA), platelet-derived growth factor (PDGF) (P-GF.44C; dilution 1:800, Novocastra, Newcastle-upon-Tyne, UK), platelet-derived growth factor 

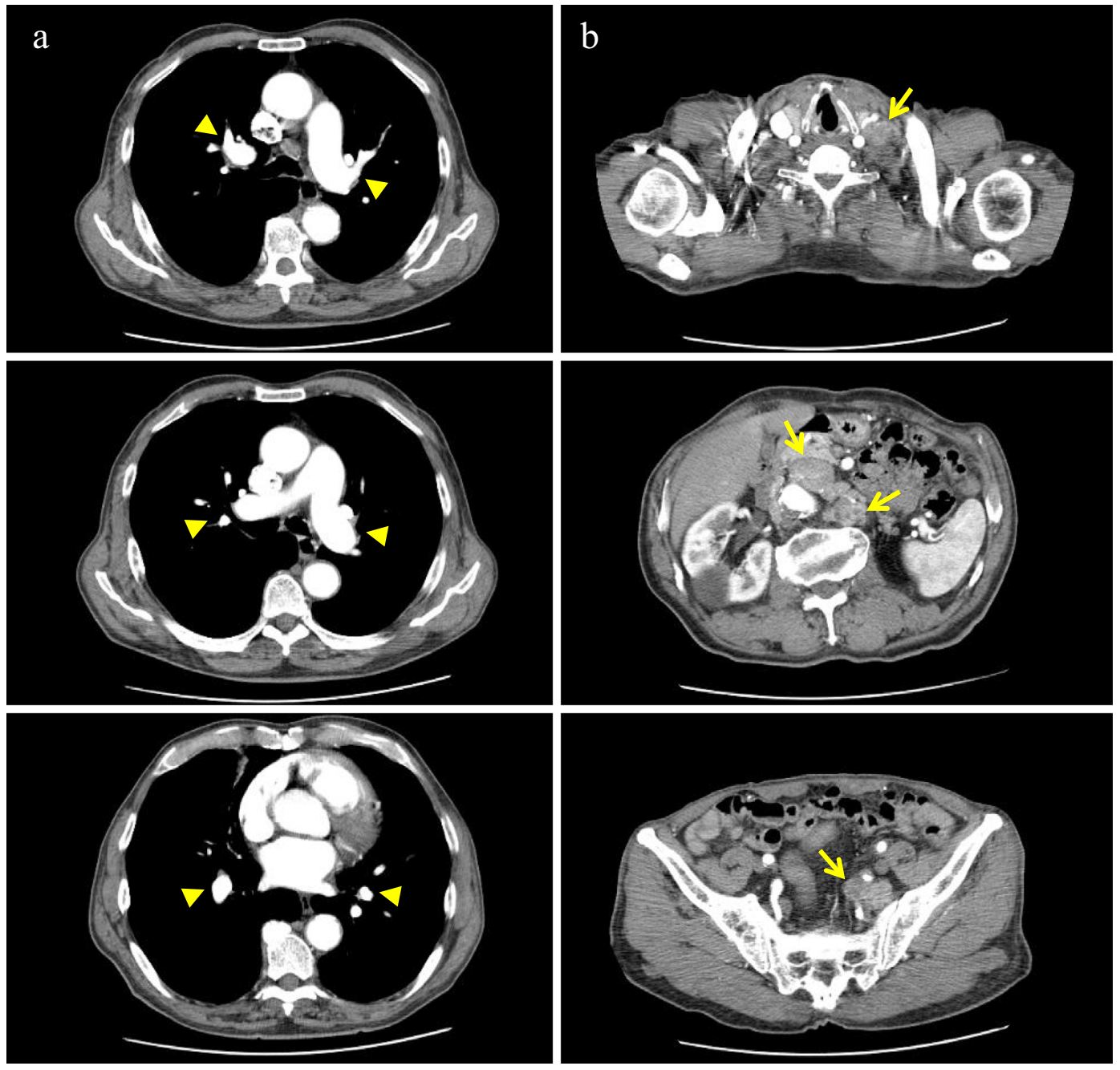

Figure 2. Contrast-enhanced computed tomography. a) Thrombi or tumor emboli were not found in the pulmonary arteries. Intact pulmonary arteries are indicated by yellow arrowheads. b) Lymphadenopathy of the supraclavicular, para-aortic, and left pelvic lymph nodes was observed, suggesting multiple lymph node metastases. Lymphadenopathy is indicated by the yellow arrows.
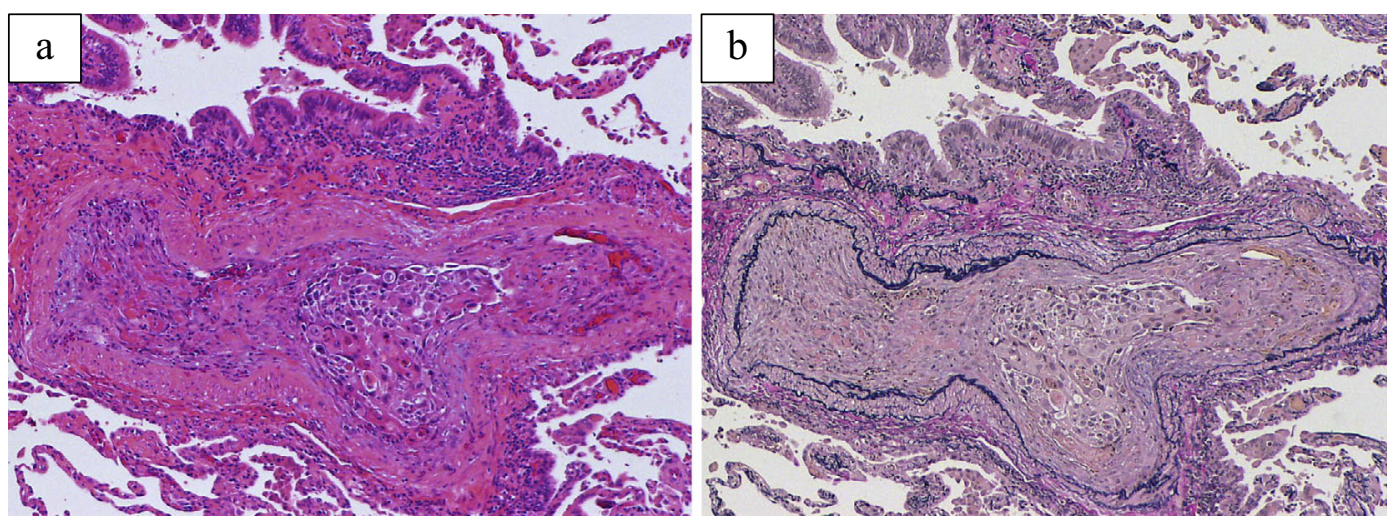

Figure 3. Histopathological findings in the small pulmonary arteries. a) Hematoxylin and Eosin staining (original magnification 40x). b) Elastica van Gieson staining (original magnification 40x). Tumor emboli of urothelial carcinoma with squamous differentiation were observed in the small pulmonary arteries. Marked intimal fibrocellular proliferation and clot formation were also observed. These findings were consistent with pulmonary tumor thrombotic microangiopathy. 

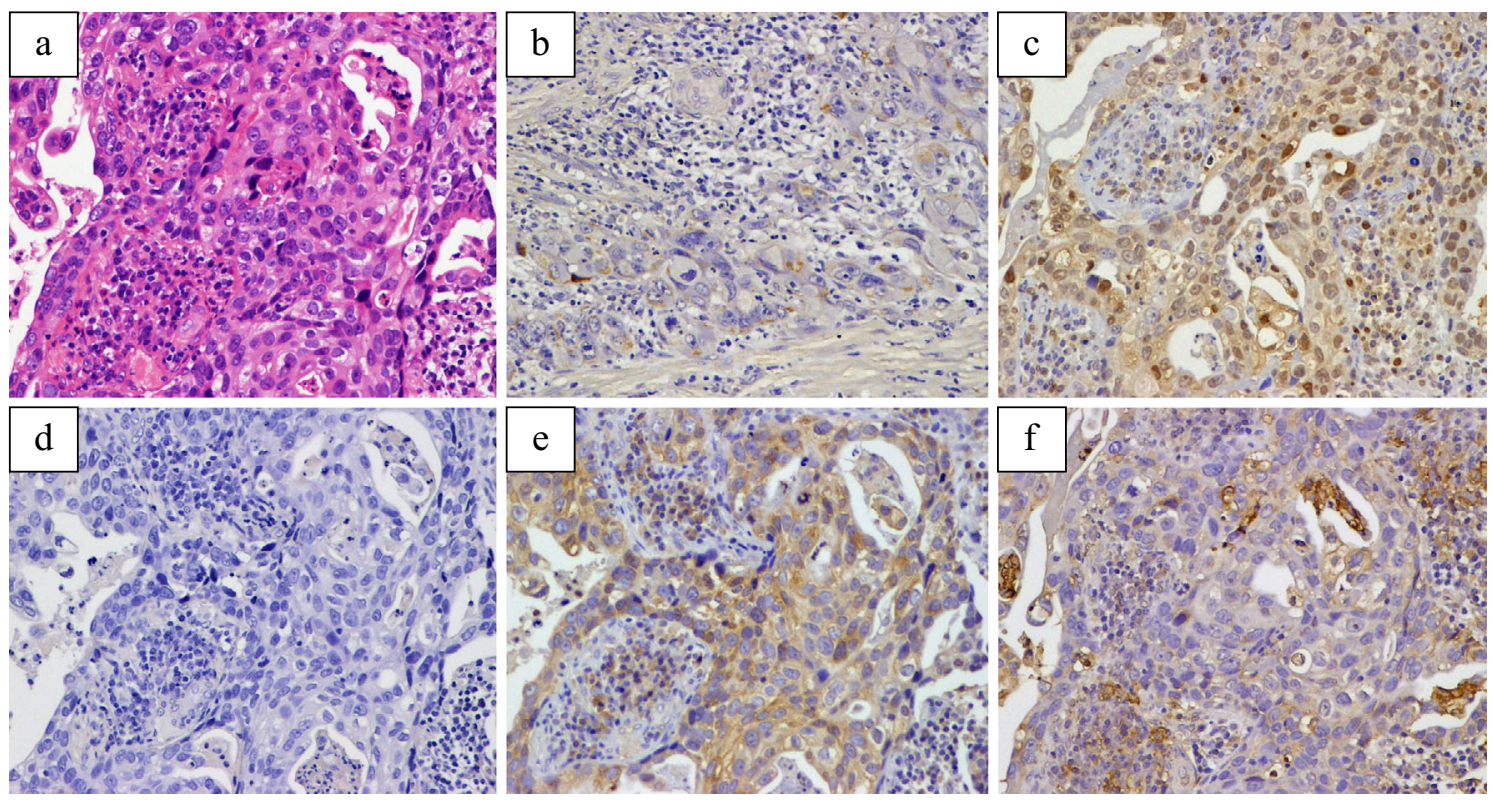

\begin{abstract}
Figure 4. Immunohistochemical findings of the primary tumor cells. a) Hematoxylin and Eosin staining (original magnification 100x). b) Staining for vascular endothelial growth factor (VEGF) (original magnification 100x). c) Staining for platelet-derived growth factor (PDGF) (original magnification 100x). d) Staining for platelet-derived growth factor receptor $\beta$ (PDGFR $\beta$ ) (original magnification $100 \times$ ). e) Staining for osteopontin (original magnification 100x). f) Staining for fibroblast growth factor 2 (FGF2) (original magnification 100x). Focal expression of VEGF, PDGF and FGF2, and diffuse expression of osteopontin were found in the primary tumor cells. However, PDGFR $\beta$ was not expressed.
\end{abstract}

receptor $\beta$ (PDGFR $\beta$ ) (rabbit polyclonal; dilution 1:1,000, Santa Crus Biotechnology, Santa Crus, USA), fibroblast growth factor 2 (FGF2) (rabbit polyclonal; dilution 1:100, Santa Crus Biotechnology), and osteopontin (OP3N, dilution 1:1,000, Novocastra). In the primary tumor cells, the focal expression of VEGF, PDGF and FGF2 was observed. Diffuse expression of osteopontin was also detected, although PDGFR $\beta$ was not expressed in the primary tumor cells (Fig. 4). In the metastatic cells, the focal expression of VEGF and PDGF, as well as the diffuse expression of osteopontin, was observed. VEGF expression was stronger in the metastatic cells than in the primary tumor cells. PDGF and osteopontin were also expressed in the proliferating intimal and vascular smooth muscle cells in the PTTM lesions. However, PDGFR $\beta$ and FGF2 were not expressed in the metastatic cells (Fig. 5).

\section{Discussion}

PTTM is thought to arise from the direct attachment of tumor cells to the endothelium of the pulmonary vascular system and the development of local thrombosis at the surface of tumor emboli, causing the release of inflammatory mediators. These mediators induce fibrocellular intimal proliferation of small pulmonary arteries and arterioles, leading to diffuse narrowing of the pulmonary artery and an increase in vascular resistance (1). For these reasons, patients with PTTM often present with progressive dyspnea, severe pul- monary hypertension, and right cardiac failure (5).

Previous studies have suggested the development of PTTM to be associated with certain molecules produced by tumor cells, including VEGF, PDGF, and osteopontin. VEGF is known as an endothelial cell-specific angiogenic mitogen involved in the proliferation of endothelial cells (6), and several PTTM cases that showed VEGF expression in tumor cells have been reported (6-8). PDGF is a key mediator of proliferation and migration of smooth muscle cells and fibroblasts (9), and osteopontin is known to promote the adhesion, migration, and proliferation of fibroblasts, vascular endothelial cells, and smooth muscle cells (10). Takahashi et al. reported a case of PTTM caused by gastric carcinoma that showed immunoreactivity to PDGF and osteopontin in tumor cells and fibromuscular intimal cells in the PTTM lesions. They suggested that an interrelationship between PDGF and osteopontin may be involved in the pathogenesis of PTTM (11). With respect to PTTM associated with urothelial carcinoma, a single case that included an investigation of these molecules was published; VEGF and PDGF were expressed in the primary tumor cells whereas PDGFR $\alpha$ and PDGFR $\beta$ were not expressed. Moreover, VEGF, PDGF, PDGFR $\alpha$, and PDGFR $\beta$ were not expressed in the metastatic cells in the PTTM lesions, although the authors reported the probability of diminished antigen activity caused by the period of formalin fixation (3). Therefore, ours is the first case of PTTM associated with urothelial carcinoma that showed immunoreactivity to VEGF, PDGF, and 

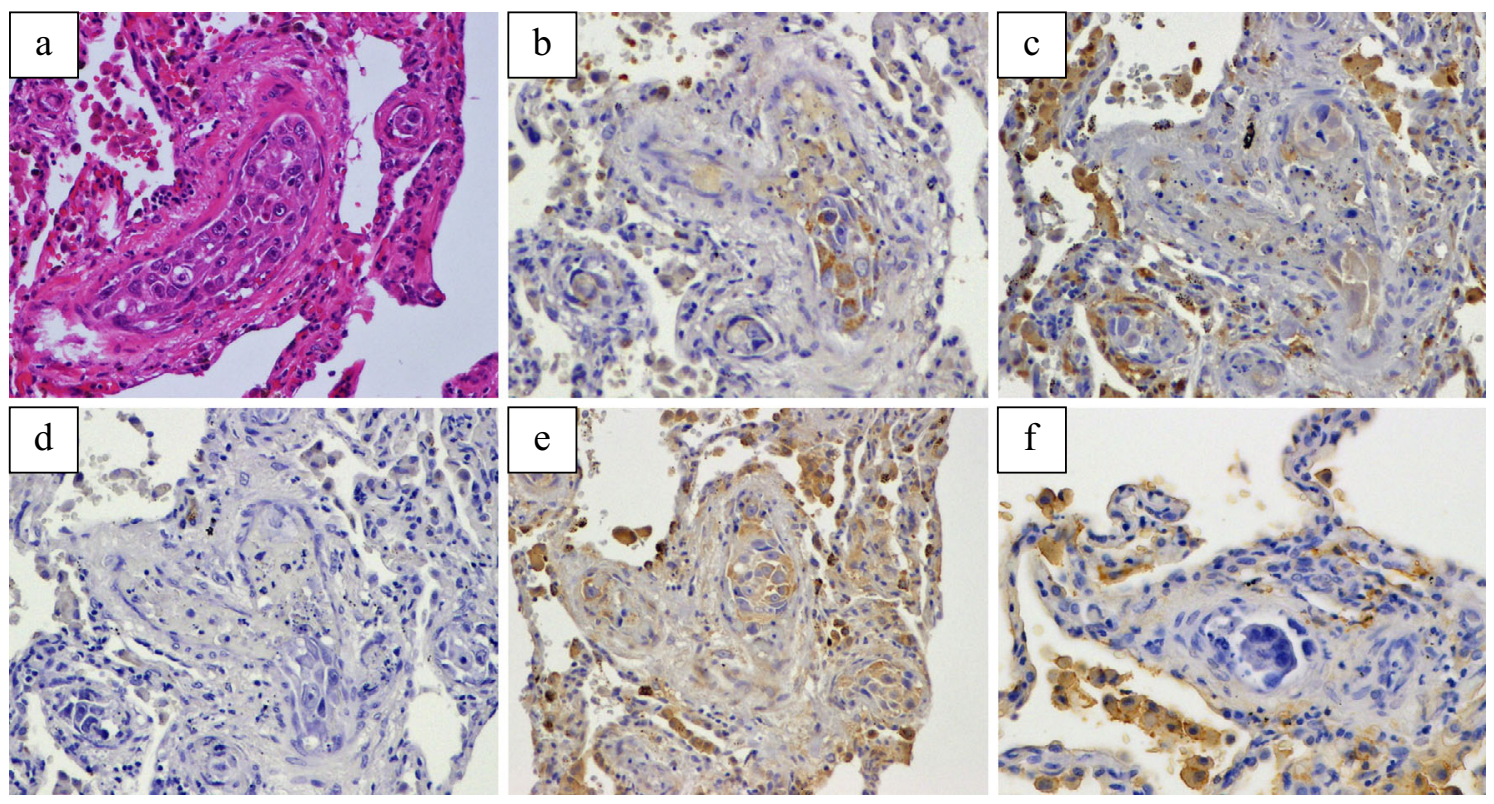

Figure 5. Immunohistochemical findings of the metastatic cells in the small pulmonary arteries. a) Hematoxylin and Eosin staining (original magnification 100x). b) Staining for vascular endothelial growth factor (VEGF) (original magnification 100x). c) Staining for platelet-derived growth factor (PDGF) (original magnification 100x). d) Staining for platelet-derived growth factor receptor $\beta$ (PDGFR $\beta$ ) (original magnification 100x). e) Staining for osteopontin (original magnification 100x). $f$ ) Staining for fibroblast growth factor 2 (FGF2) (original magnification 600x). Focal expression of VEGF and PDGF, and diffuse expression of osteopontin, were observed in the metastatic cells. PDGF and osteopontin were also expressed in the proliferating intimal cells and vascular smooth muscle cells. However, PDGFR $\beta$ and FGF2 were not expressed in the metastatic cells.

osteopontin in both the primary and metastatic tumor cells. We therefore postulate that VEGF, PDGF, and osteopontin may be related to the onset or development of PTTM caused by urothelial carcinoma.

With respect to PDGFR, two previous cases of PTTM caused by gastric carcinoma were reported to show expression of PDGFR $\alpha$ and PDGFR $\beta$ in the PTTM lesions $(12,13)$. Additionally, Abe et al. reported eight cases of PTTM caused by gastric carcinoma that showed FGF2 expression (14). In the present case, neither PDGFR $\beta$ nor FGF2 was detected in the PTTM lesions. Given the previously described precedence (3), we suspect that the antigenic activities of these proteins may have been diminished because of the extended period of formalin fixation.

An antemortem diagnosis and successful treatment of PTTM is extremely difficult. However, a case of PTTM caused by colorectal adenocarcinoma was previously reported to have been successfully treated with a combination of imatinib (a PDGFR antagonist) and bevacizumab (a VEGF receptor inhibitor) (15). In the present case, the expression of VEGF, PDGF, and osteopontin suggests that patients with PTTM caused by urothelial carcinoma also have the potential to be treated effectively with these moleculartargeted agents, despite the fact that PDGFR $\beta$ was not detected and PDGFR $\alpha$ expression was not investigated. Furthermore, in cases of PTTM, the various molecules that are expressed in tumor cells should be examined, as this may offer additional clues regarding the mechanism of PTTM development and subsequently lead to successful treatment with molecular-targeted agents.

The authors state that they have no Conflict of Interest (COI).

\section{References}

1. von Herbay A, Illes A, Waldherr R, Otto HF. Pulmonary tumor thrombotic microangiopathy with pulmonary hypertension. Cancer 66: $587-592,1990$.

2. Uruga H, Fujii T, Kurosaki A, et al. Pulmonary tumor thrombotic microangiopathy: a clinical analysis of 30 autopsy cases. Intern Med 52: 1317-1323, 2013.

3. Hirano H, Ichibori H, Kizaki T, et al. Pulmonary tumor thrombotic microangiopathy showing aggressive course after transurethral resection of urinary bladder: an autopsy case report. Med Mol Morphol 45: 238-242, 2012.

4. Marumo S, Sakaguchi M, Teranishi T, Higami Y, Koshimo Y, Kato M. Pulmonary tumor thrombotic microangiopathy induced by ureteral carcinoma: a necropsy case report. Case Rep Oncol 7: 605-610, 2014.

5. Okubo Y, Wakayama M, Kitahara K, et al. Pulmonary tumor thrombotic microangiopathy induced by gastric carcinoma: morphometric and immunohistochemical analysis of six autopsy cases. Diagn Pathol 6: 27, 2011.

6. Chinen K, Tokuda Y, Fujiwara M, Fujioka Y. Pulmonary tumor thrombotic microangiopathy in patients with gastric carcinoma: an analysis of 6 autopsy cases and review of the literature. Pathol Res Pract 206: 682-689, 2010. 
7. Chinen K, Kazumoto T, Ohkura Y, Matsubara O, Tsuchiya E. Pulmonary tumor thrombotic microangiopathy caused by a gastric carcinoma expressing vascular endothelial growth factor and tissue factor. Pathol Int 55: 27-31, 2005.

8. Chinen K, Fujino T, Horita A, Sakamoto A, Fujioka Y. Pulmonary tumor thrombotic microangiopathy caused by an ovarian cancer expressing tissue factor and vascular endothelial growth factor. Pathol Res Pract 205: 63-68, 2009.

9. Heldin $\mathrm{CH}$, Westermark B. Mechanism of action and in vivo role of platelet-derived growth factor. Physiol Rev 79: 1283-1316, 1999.

10. Denhardt DT, Noda M, O'Regan AW, Pavlin D, Berman JS. Osteopontin as a means to cope with environmental insults: regulation of inflammation, tissue remodeling, and cell survival. J Clin Invest 107: 1055-1061, 2001.

11. Takahashi F, Kumasaka T, Nagaoka T, et al. Osteopontin expres- sion in pulmonary tumor thrombotic microangiopathy caused by gastric carcinoma. Pathol Int 59: 752-756, 2009.

12. Minatsuki S, Miura I, Yao A, et al. Platelet-derived growth factor receptor-tyrosine kinase inhibitor, imatinib, is effective for treating pulmonary hypertension induced by pulmonary tumor thrombotic microangiopathy. Int Heart J 56: 245-248, 2015.

13. Yokomine T, Hirakawa H, Ozawa E, Shibata K, Nakayama T. Pulmonary thrombotic microangiopathy caused by gastric carcinoma. J Clin Pathol 63: 367-369, 2010.

14. Abe H, Hino R, Fukayama $M$. Platelet-derived growth factor-A and vascular endothelial growth factor-C contribute to the development of pulmonary tumor thrombotic microangiopathy in gastric cancer. Virchows Arch 462: 523-531, 2013.

15. Higo K, Kubota K, Takeda A, Higashi M, Ohishi M. Successful antemortem diagnosis and treatment of pulmonary tumor thrombotic microangiopathy. Intern Med 53: 2595-2599, 2014.

(C) 2016 The Japanese Society of Internal Medicine http://www.naika.or.jp/imonline/index.html 